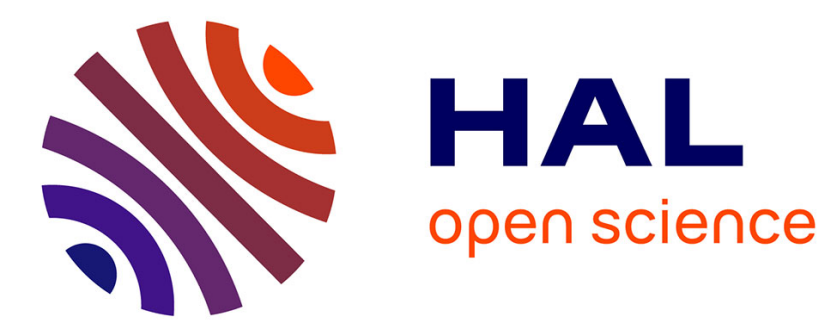

\title{
Transient radiative tranfer in the grey case: well-balanced and asymptotic-preserving schemes built on Case's elementary solutions \\ Laurent Gosse
}

\section{- To cite this version:}

Laurent Gosse. Transient radiative tranfer in the grey case: well-balanced and asymptotic-preserving schemes built on Case's elementary solutions. Journal of Quantitative Spectroscopy and Radiative Transfer, 2011, 112, pp.1995-2012. 10.1016/j.jqsrt.2011.04.003 . hal-00540294

\section{HAL Id: hal-00540294 \\ https://hal.science/hal-00540294}

Submitted on 26 Nov 2010

HAL is a multi-disciplinary open access archive for the deposit and dissemination of scientific research documents, whether they are published or not. The documents may come from teaching and research institutions in France or abroad, or from public or private research centers.
L'archive ouverte pluridisciplinaire HAL, est destinée au dépôt et à la diffusion de documents scientifiques de niveau recherche, publiés ou non, émanant des établissements d'enseignement et de recherche français ou étrangers, des laboratoires publics ou privés. 


\title{
Transient radiative tranfer in the grey case: well-balanced and asymptotic-preserving schemes built on Case's elementary solutions
}

\author{
Laurent Gosse*
}

November 26, 2010

\begin{abstract}
An original well-balanced (WB) Godunov scheme relying on an exact Riemann solver involving a nonconservative (NC) product is developed in order to solve accurately the time-dependent one-dimensional radiative transfer equation in the discrete-ordinates approximation with an arbitrary even number of velocities. The collision term is thus concentrated onto a discrete lattice by means of Dirac masses; this induces steady jump relations across with the stationary problem is solved by taking advantage of the method of elementary solutions mainly developed by Case, Zweifel and Cercignani. This approach produces a rather simple scheme that compares advantageously to standard existing upwind schemes, especially for the decay in time toward a Maxwellian distribution. It is possible to reformulate this scheme in order to handle properly the parabolic scaling in order to generate a so-called asymptotic-preserving (AP) discretization for which the consistency with the diffusive approximation holds independently of the computational grid. Several numerical results are displayed to show the realizability and the efficiency of the method.
\end{abstract}

\section{Introduction}

This paper is a continuation of the former works $[22,23,24]$; it aims at pushing toward more complex kinetic models the development of numerical schemes satisfying both the well-balanced (WB) and the asymptotic-preserving (AP) criteria in hyperbolic and parabolic scalings, respectively. More precisely, we first derive a WB scheme which solves the Cauchy problem for a simple model of "grey" radiative transfer:

$$
\partial_{t} f+\xi \partial_{x} f=\frac{c}{2} \int_{-1}^{1} f\left(t, x, \xi^{\prime}\right) d \xi^{\prime}-f, \quad \xi \in[-1,1], x \in \mathbb{R}, t>0 .
$$

*IAC-CNR "Mauro Picone" (sezione di Bari), Via Amendola 122/D, 70126 Bari (Italy) 1.gosse@ba.iac.cnr.it 
This model is also relevant for neutron transport: in this case, the parameter $c \in[0,1]$ stands for the mean number of secondary neutrons obtained by both fission and scattering per collision. It is of course completed by initial conditions:

$$
f(t=0, x, \xi)=f^{0}(x, \xi), \quad \xi \in[-1,1], x \in \mathbb{R} .
$$

A central feature of kinetic problems of the type (1) is to admit a so-called "diffusive approximation": let us define the macroscopic density and flux as

$$
\varrho(t, x)=\int_{-1}^{1} f(t, x, \xi) d \xi, \quad J(t, x)=\int_{-1}^{1} \xi f(t, x, \xi) d \xi
$$

then, for $c=1$, it can be shown rigorously that upon rescaling (1) as follows,

$$
\varepsilon^{2} \partial_{t} f+\varepsilon \xi \partial_{x} f=\frac{1}{2} \int_{-1}^{1} f\left(t, x, \xi^{\prime}\right) d \xi^{\prime}-f, \quad 0<\varepsilon \ll 1,
$$

in the limit $\varepsilon \rightarrow 0$, the following diffusion holds $[3,38]$ :

$$
\partial_{t} \varrho-\frac{1}{3} \partial_{x x} \varrho=0, \quad x \in \mathbb{R}, t>0 .
$$

In particular, one obtains in this case:

$$
f(t, x, \xi) \equiv \frac{1}{2} \varrho(t, x), \quad J(t, x)=-\frac{1}{3} \partial_{x} \varrho(t, x) .
$$

Our goal in this paper is to derive a numerical scheme for the time-dependent equation (1) in the discrete-ordinates approximation [44]; it is meant to be consistent with both the large-time asymptotic behavior (see e.g. [2]) and with the diffusive approximation (3) when applied to the parabolic scaling (2). Up to now, this program has been completed only for 2-velocities models [22, 23]. We stress that these algorithms have been used in several meaningful areas of application: see e.g. [6, 8, 9, 28].

Two complementary methodologies emerged for deriving numerical schemes approximating nonhomogeneous hyperbolic problems: the well-balanced schemes [25] roughly asking for an enhanced consistency with the long-time behavior of the original equation, and the asymptotic-preserving schemes [33, 31] which ensure that the limiting process $\varepsilon \rightarrow 0$ leading to the diffusive approximation still holds at the discrete level independently of the size of the computational grid. For discrete velocity models, it has been shown rigorously in $[22,23,24]$ that the asymptotic-preserving property is actually a consequence of the well-balanced when one implements it through a Godunov scheme relying onto an exact Riemann solver. More precisely, the path is to pass from the Cauchy problem for (1) to the homogeneous (but non-conservative) one (15). This reformulation, which concentrates the collision term onto a discrete lattice related to the computational grid, allows to treat the right-hand side by means of Rankine-Hugoniot type jump relations across which one follows the solution of the stationary problem for (1), see (4). Clearly, the stiffness issue disappear because multiplying 
the right-hand side by any big number boils simply down to going much farer on these steady-state curves as a rescaling in $x$ easily shows.

More interestingly, it has been first shown in [22] that these non-conservative jump relations can be divided into 2 qualitatively different terms: the first one is $O(1)$ and corresponds to the strong relaxation onto the Maxwellian distribution and the second one is $O(\varepsilon)$ and contains the diffusive terms which allow to be consistent with (3) as $\varepsilon \rightarrow 0$ with $\varepsilon \ll \Delta x$ and $\Delta x>0$. Clearly, in this regime, the time-step has to meet with the usual parabolic CFL condition, that is $\Delta t=O\left(\Delta x^{2}\right)$. Efforts have been made in [23, 24] (and more recently in [21] for a model of chemotaxis movement) to extend this framework toward more complex kinetic models: we present in this paper an original Godunov WB scheme for (1) which is built on the classical theory of so-called "elementary solutions" introduced by Case [10] and developed by various authors [11, 12, 13, 14]. As it was the case for simpler discrete-velocity models, this WB scheme can be reformulated in order to handle the diffusive scaling (2) and be consistent as $\varepsilon \rightarrow 0$ with the limiting equation (3).

Another feature emerged while studying this type of numerical schemes: namely, it appears that conventional upwind schemes for the linear equation (1) generally do not stabilize onto the exact Maxwellian distribution as $t \rightarrow+\infty$ with $\Delta x>0$ fixed despite the fact that the residues plunge to zero. Ripples keep on existing when ones visualizes the numerical kinetic density $f(t, x, \xi)$ in the plane $x, \xi$ even if the residues are of the order of $10^{-7}$ : see Figure 7 on the right. In sharp contrast, our resulting well-balanced schemes stabilize nicely with time and, apart from the low velocities $|\xi|<0.2$, the kinetic density $f$ is truly constant in the $\xi$ variable. Meaningful steps of this time stabilization process for the equation (1) inside a box with reflecting boundary conditions are shown in Figure 5. This dynamic is much more consistent with the asymptotic behavior $[2,35]$ of (1) than the one coming from more conventional upwind discretizations.

Accordingly, this paper is organized as follows: in $\S 2$, we recall both the Case's method of elementary solutions with the completeness results from [13, $26,34]$ and the ADO method of $[5,40]$. Later, the well-balanced scheme for rarefied regime is derived relying on these solutions of the stationary equation, in the dissipative case $c<1$ in $\S 3.1$ and in the conservative case $c=1$ in $\S 3.2$; a comparison with a classical time-splitting scheme is presented in $\S 3.3$. The reformulation as a AP scheme is derived in $\S 4.1$; in $\S 4.2$, we show the relation existing with an earlier scheme in [24]. Numerical results for small values of $\varepsilon<\Delta x$ are displayed in $\S 4.3$ where the consistency with (3) can be seen. Lastly, $\S 5$ contains some concluding remarks.

Let us close this introduction with some bibliographic comments. Elementary solutions (also referred to as "spectral Green functions" [17]) have been used in a numerical context for steady-state radiative transfer problems (involving coupling) in $[32,45]$. The discrete-ordinates method, consisting in discretizing the velocity variable according to a Gaussian quadrature rule is classical [44] and has been studied in parabolic regime in e.g. [30]. The method of resolution developed by Case is by no means limited to radiative transfer problems: it has 
been extended to BGK models by Cercignani in [12], see the survey in Chapter 6 of [14]. Ch. Dalitz [16, 18] extended it further to Boltzmann models of charged particles for which the collision term is not a self-adjoint operator. More generally, this method can be recast into the framework of exponentially dichotomous operators: see the Chapter 5 in the book [42]. Spurious long-time behavior of standard upwind numerical schemes in a particular context has been detected in [27] where stabilization onto stationary regimes with very big macroscopic fluxes have been observed. Obviously, for the 2-velocity model considered in [27], a non-zero macroscopic flux signals that the stationary regime isn't Maxwellian.

\section{The method of Case's elementary solutions}

\subsection{Continuous solution of the steady-state problem}

In his seminal paper, Case [10] considers the following equation:

$$
\xi \partial_{x} f(x, \xi)+f(x, \xi)=\frac{c}{2} \int_{-1}^{1} f\left(x, \xi^{\prime}\right) d \xi^{\prime}, \quad c \in[0,1], x \in \mathbb{R} .
$$

In order to solve (4), his method stems on expanding $f$ in terms of a set of eigenfunctions; invariance by translation suggests the separation of variables:

$$
f(x, \xi)=\psi(x) \varphi(\xi) .
$$

Plugging into (4) and dividing by $f$ leads to

$$
\frac{\psi^{\prime}(x)}{\psi(x)}=-\frac{1}{\xi}+\frac{c}{2 \xi \varphi(\xi)} \int_{-1}^{1} \varphi\left(\xi^{\prime}\right) d \xi^{\prime}
$$

Both sides of the equation must be equal to the constant $-\frac{1}{\nu}$ hence:

$$
\psi_{\nu}(x)=\exp (-x / \nu), \quad\left(1-\frac{\xi}{\nu}\right) \varphi_{\nu}(\xi)=\frac{c}{2} \int_{-1}^{1} \varphi\left(\xi^{\prime}\right) d \xi^{\prime} \equiv \frac{c}{2} .
$$

The first step is to find the eigenmodes $\nu$ such that both the preceding equations admit solutions. The normalization of the integral term allows to rewrite the equation on $\varphi_{\nu}$ as

$$
\left(1-\frac{\xi}{\nu}\right) \varphi_{\nu}(\xi)=\frac{c}{2}
$$

which highlights the two cases to examine, namely $\nu \in[-1,1]$ and $\nu \notin[-1,1]$.

1. $\nu \notin[-1,1]$ : this corresponds to the discrete part of the spectrum. The denominator never vanishes thus one can safely write:

$$
\varphi_{\nu}(\xi)=\frac{c}{2}\left(1-\frac{\xi}{\nu}\right)^{-1}
$$


In order to meet with the normalization of the integral term, the following condition should hold:

$$
\Lambda(\nu)=1-\frac{c \nu}{2} \log \left(\frac{\nu+1}{\nu-1}\right)=0 .
$$

For $c<1,2$ real roots exist for $\Lambda$, usually denoted $\pm \nu_{0}$; hence,

$$
\varphi_{ \pm \nu_{0}}(\xi)=\frac{c}{2}\left(1 \mp \frac{\xi}{\nu_{0}}\right)^{-1}
$$

2. $\nu \in[-1,1]$ : let $P V$ stand for the Cauchy principal value and $\delta$ for the Dirac mass in zero. Generalized solutions of (5) read now:

$$
\left.\varphi_{\nu}(\xi)=\frac{c}{2} P V\left(1-\frac{\xi}{\nu}\right)^{-1}+\lambda(\nu) \delta(\xi-\nu), \quad \nu \in\right]-1,1[,
$$

for which the normalization condition of the collision term leads to

$$
\lambda(\nu)=1-\frac{c \nu}{2} P V \int_{-1}^{1} \frac{d \xi}{\nu-\xi}=1-\frac{c \nu}{2} \log \left(\frac{\nu+1}{\nu-1}\right) .
$$

Consequently, to any value of $\nu \in]-1,1$ [ can be associated a "generalized eigenfunction" of the type (6).

This computation should be completed by a completeness assertion: indeed, it is proved in [11] that any reasonable function $f(x, \xi)$ can be expanded as follows:

$$
f(x, \xi)=a_{ \pm} \frac{c}{2}\left(1 \mp \frac{\xi}{\nu_{0}}\right)^{-1} \exp \left(\mp x / \nu_{0}\right)+\int_{-1}^{1} A(\nu) \varphi_{\nu}(\xi) \exp (-x / \nu) d \nu
$$

More precise completeness statements are available in [14] (pp. 291/2) or [34]. The case $c=1$ is critical for these computations: it is explained in the Appendix F of [11] (see also [42] p.98) that the discrete part of the spectrum has to be modified as follows:

$$
f(x, \xi)=\alpha+\beta x+\int_{-1}^{1} A(\nu) \varphi_{\nu}(\xi) \exp (-x / \nu) d \nu .
$$

For $c=1$, discrete eigenvalues are unbounded: more details on this situation are studied in [43]. From [13] (page 610) comes a result directly concerned with (4): the set of generalized eigenfunctions is complete for functions $f$ such that:

1. $\xi \mapsto f(x, \xi)$ is Hölderian in any closed subinterval of $(-1,1)$,

2. $\xi \mapsto f(x, \xi)\left(1-\xi^{2}\right)^{\gamma}$ is Hölderian in $[-1,1](0<\gamma<1)$.

Extensions to "generalized kinetic equations" $T \partial_{x} f(x, \xi)+A f=0$ in Hilbert space for $T$ bounded self-adjoint and $A$ possibly unbounded, self-adjoint and Fredholm are to be checked in [26]. The orthogonality of the generalized eigenfunctions is easily proved in the original paper by Case (see Theorem I in [10]):

$$
\int_{-1}^{1} \xi \varphi_{\nu}(\xi) \varphi_{\nu^{\prime}}(\xi) d \xi=0, \quad \nu \neq \nu^{\prime}
$$




\subsection{The Analytical Discrete-Ordinate (ADO) method}

The Case's method of elementary functions has been extended by many authors, see e.g. $[11,12,13,14,16,17,18,34]$. For numerical purposes, it has been introduced under the name "Analytical Discrete-Ordinate method" in [5]. Roughly speaking, it consists in first, introducing a $N$-point Gaussian quadrature on the interval $(0,1)$ given by the following points and weights:

$$
\boldsymbol{\xi}=\left(\xi_{1}, \xi_{2}, \ldots, \xi_{N}\right) \in(0,1)^{N}, \quad \boldsymbol{\omega}=\left(\omega_{1}, \ldots, \omega_{N}\right) \in \mathbb{R}^{+}
$$

Then, it computes a vector of eigenmodes $\boldsymbol{\nu} \in\left(\mathbb{R}^{+}\right)^{N}$ which is an approximation of both the discrete and continuous part of the spectrum derived in the preceding subsection. Last, it determines the coefficients of the generalized eigenfunctions out of the given inflow boundary conditions. Let us rewrite (4) as follows:

$$
\xi \partial_{x} f(x, \xi)+f(x, \xi)=\frac{c}{2} \int_{0}^{1} f\left(x, \xi^{\prime}\right)+f\left(x,-\xi^{\prime}\right) d \xi^{\prime}, \quad x \in\left[-x_{0}, x_{0}\right]
$$

with $\xi \in[-1,1]$ and supplemented by inflow boundary conditions:

$$
f\left( \pm x_{0}, \mp|\xi|\right)=F_{L / R}(\xi), \quad \xi \in(0,1] .
$$

Analogously with the continuous case, the separation variable $\nu$ is introduced:

$$
f(x, \xi)=\varphi(\nu, \xi) \exp (-x / \nu) .
$$

Plugging into (10) and taking the quadrature rule into account yields:

$$
\left(1 \mp \frac{\xi_{k}}{\nu_{k}}\right) \varphi\left(\nu, \pm \xi_{k}\right)=\frac{c}{2} \sum_{\ell=1}^{N} \omega_{\ell}\left(\varphi\left(\nu, \xi_{\ell}\right)+\varphi\left(\nu,-\xi_{\ell}\right)\right), \quad k \in\{1, \ldots, N\} .
$$

It is at this level that a trick is used in order to reduce the cost of this eigenvalue problem: let us denote $\Phi_{ \pm}(\nu)=\left(\varphi\left(\nu, \pm \xi_{k}\right)\right)_{k \in\{1, \ldots, N\}}$ and $I d$ the identity matrix of $\mathbb{R}^{N}$. By using the same notation for a vector in $\mathbb{R}^{N}$ and its corresponding $N \times N$ diagonal matrix, it comes:

$$
\pm \frac{1}{\nu} \xi \Phi_{ \pm}(\nu)=\left(I d-\frac{c}{2} \omega\right) \Phi_{ \pm}(\nu)-\frac{c}{2} \omega \Phi_{\mp}(\nu)
$$

Barichello, Siewert and Wright $[5,40]$ now observe that, upon defining $S(\nu)=$ $\boldsymbol{\xi}\left(\Phi_{+}(\nu)+\Phi_{-}(\nu)\right) \in \mathbb{R}^{N},(12)$ reduces to:

$$
\boldsymbol{\xi}^{-1}(I d-c \boldsymbol{\omega}) \boldsymbol{\xi}^{-1} S(\nu)=\frac{1}{\nu^{2}} S(\nu) .
$$

This problem can be recast under a very tractable one, to which standard divideand-conquer methods [15] can be applied; indeed, by multiplying by the diagonal $N \times N$ matrix $T=\operatorname{diag}\left(\sqrt{\omega_{k}}\right)$, it comes

$$
\left(\boldsymbol{\xi}^{-2}-c z z^{T}\right) X(\nu)=\frac{1}{\nu^{2}} X(\nu)
$$


where:

$$
z=\operatorname{diag}\left(\frac{\sqrt{\omega_{k}}}{\xi_{k}}\right)=\sqrt{\boldsymbol{\omega}} \boldsymbol{\xi}^{-1}, \quad X(\nu)=T S(\nu) .
$$

The eigenproblem is known to possess numerous "good properties" as explained in [40]; in particular, since the components of $z$ never vanish, we have the interlacing repartition,

$$
0<\xi_{1}<\nu_{1}<\xi_{2}<\nu_{2}<\ldots<\xi_{N}<\nu_{N} \notin(0,1]
$$

Clearly, $\nu_{N}$ stand for the discrete part of the spectrum and thus can become very big when $c \rightarrow 1$; in the limit $c=1$, we have a degeneracy at infinity. Finally, we recall the normalization of the $N$ eigenvectors:

$$
\sum_{\ell=1}^{N} \omega_{\ell}\left(\varphi\left(\nu_{k}, \xi_{\ell}\right)+\varphi\left(\nu_{k},-\xi_{\ell}\right)\right)=1, \quad k \in\{1, \ldots, N\} .
$$

\section{Well-balanced scheme based on exact Riemann solver for kinetic regime}

Starting from here, we define a space/time computational grid determined by a time step $\Delta t>0$ and the uniform width of the cells $\Delta x>0$ such that the CFL condition holds: $\Delta t \leq \Delta x$. We obtain:

$$
x_{j}=j \Delta x, t^{n}=n \Delta t, C_{j}=\left(x_{j-\frac{1}{2}}, x_{j+\frac{1}{2}}\right), \quad j \in \mathbb{Z}, n \in \mathbb{N} .
$$

Then we introduce approximate values as follows:

$$
f_{j}^{n}\left( \pm \xi_{k}\right) \simeq f\left(t^{n}, x_{j}, \pm \xi_{k}\right)
$$

where $\xi_{k}$ still refers to the Gaussian quadrature rule (9). The general methodology of well-balanced schemes stems on localizing the source terms of hyperbolic equations onto a discrete lattice; presently, it consists in passing from (1) to:

$$
\partial_{t} f+\xi \partial_{x} f=\sum_{j \in \mathbb{Z}} \Delta x\left(\frac{c}{2} \int_{-1}^{1} f\left(t, x, \xi^{\prime}\right) d \xi^{\prime}-f\right) \delta\left(x-x_{j+\frac{1}{2}}\right) .
$$

The consistency with the original problem as $\Delta x \rightarrow 0$ is the consequence of the simple observation:

$$
\sum_{j \in \mathbb{Z}} \Delta x \delta\left(x-x_{j+\frac{1}{2}}\right) \rightarrow 1 .
$$

More details about consistency for hyperbolic systems of balance laws are to be found in [1]. For discontinuous solutions, the right-hand side of (15) has become a non-conservative (NC) product and should be defined carefully. It has been rigorously shown in [23] (see §2.1) that in the simpler case of a discrete velocity model, the localization process yields a BV-bound on the corresponding 
sequence of solutions. The NC product can therefore be defined as a weak limit in the theory of [36]. It induces a stationary contact discontinuity across which holds a jump relation following the integral curves of the steady-state equation (4). This is the reason why the steady-state problem has been studied in the preceding section. The macroscopic density and flux are defined according to the quadrature rule introduced while setting up the ADO method:

$$
\varrho_{j}^{n}=\sum_{k=1}^{N} \omega_{k}\left(f_{j}^{n}\left(\xi_{k}\right)+f_{j}^{n}\left(-\xi_{k}\right)\right), \quad J_{j}^{n}=\sum_{k=1}^{N} \omega_{k} \xi_{k}\left(f_{j}^{n}\left(\xi_{k}\right)-f_{j}^{n}\left(-\xi_{k}\right)\right) .
$$

\subsection{Dissipative case $0<c<1$ (absorption and scattering)}

We aim at constructing our well-balanced scheme as a Godunov scheme relying on the exact Riemann solver for the NC problem (15). We recall that this formulation makes the problem nonlinear as it rewrites [23, 29]:

$$
\partial_{t} f+\xi \partial_{x} f-\left(\frac{c}{2} \int_{-1}^{1} f\left(t, x, \xi^{\prime}\right) d \xi^{\prime}-f\right) \partial_{x} a=0, \quad \partial_{t} a=0 .
$$

The Riemann problem for (15) consists in solving the equation for piecewise constant initial data for $\xi \in[-1,1]$ :

$$
f^{0}(x<0, \xi)=f_{\text {left }}(\xi), \quad f^{0}(x>0, \xi)=f_{\text {right }}(\xi) .
$$

Its structure is simple since $\partial_{x} a(x) \neq 0$ only at $x=0$, thus the propagating advection waves are unaffected by the $\mathrm{NC}$ product. Therefore, we are led to solve the boundary value problem for (4) inside the interval $x \in\left(-\frac{\Delta x}{2}, \frac{\Delta x}{2}\right)$ in the discrete-ordinates approximation, that is, for $\pm \xi \in \boldsymbol{\xi}$ only. The first step is clearly to determine the eigenmodes $\nu \in \mathbb{R}^{N}$ : they are valid whatever the computational point $x_{j}, t^{n}$ so they must be computed as a pre-processing step, before starting the iterations in time.

When the eigenmodes are known, we pass to the resolution of the boundaryvalue problem under the normalization (14) and the conditions (11) which can be deduced easily from the Riemann data:

$$
F_{L}(\xi>0)=f_{\text {left }}(\xi>0), \quad F_{R}(\xi<0)=f_{\text {right }}(\xi<0) .
$$

The discrete-ordinates approximation considers the following expression for the stationary solution with $\xi_{k} \in \boldsymbol{\xi}$ : (see also $\S 5.3 .1$ in [42])

$$
f\left(x, \pm \xi_{k}\right)=\frac{c}{2} \sum_{\ell=1}^{N}\left(\frac{A_{\ell} \exp \left(-\left(\frac{\Delta x}{2}+x\right) / \nu_{\ell}\right)}{1 \mp \xi_{k} / \nu_{\ell}}+\frac{B_{\ell} \exp \left(-\left(\frac{\Delta x}{2}-x\right) / \nu_{\ell}\right)}{1 \pm \xi_{k} / \nu_{\ell}}\right)
$$

Comparing with (7), it appears that the $N-1$ first terms correspond to a finite approximation of the integral term coming from the continuous spectrum (also called "damped modes") and the last one matches the discrete part. The 
coefficients vectors $\mathbf{A}=\left(A_{\ell}\right)$ and $\mathbf{B}=\left(B_{\ell}\right)$ for $\ell=1, \ldots, N$ are determined by the boundary conditions through the resolution of:

$$
M\left(\begin{array}{c}
\mathbf{A} \\
\mathbf{B}
\end{array}\right)=\frac{2}{c}\left(\begin{array}{c}
f_{\text {left }}(\boldsymbol{\xi}) \\
f_{\text {right }}(-\boldsymbol{\xi})
\end{array}\right)
$$

Using tensorial products notation, the symmetric matrix $M$ reads as follows:

$$
M=\left(\begin{array}{cc}
\left(1-\boldsymbol{\xi} \otimes \boldsymbol{\nu}^{-1}\right)^{-1} & \left(1+\boldsymbol{\xi} \otimes \boldsymbol{\nu}^{-1}\right)^{-1} \exp \left(-\frac{\Delta x}{\boldsymbol{\nu}}\right) \\
\left(1+\boldsymbol{\xi} \otimes \boldsymbol{\nu}^{-1}\right)^{-1} \exp \left(-\frac{\Delta x}{\boldsymbol{\nu}}\right) & \left(1-\boldsymbol{\xi} \otimes \boldsymbol{\nu}^{-1}\right)^{-1}
\end{array}\right)
$$

where $\exp \left(-\frac{\Delta x}{\nu}\right)$ stands for the $N \times N$ diagonal matrix of values $\exp \left(-\frac{\Delta x}{\nu_{k}}\right)$. Once again, this matrix has to be assembled and inverted once for all as a pre-processing step. By analogy with [22], we use the following notation:

$$
\tilde{f}_{L}\left(-\xi_{k}\right):=f\left(-\frac{\Delta x}{2},-\xi_{k}\right), \quad \tilde{f}_{R}\left(\xi_{k}\right):=f\left(\frac{\Delta x}{2}, \xi_{k}\right) .
$$

The uniform (in $N$ and $\Delta x$ ) invertibility of $M$ comes from the completeness theorems for Case's generalized eigenfunctions. By recalling the simple fact that the Godunov scheme consists in evaluating numerical fluxes at the interfaces of each computational cell $C_{j}$, we are now in position to write it down:

$$
\left\{\begin{array}{l}
f_{j}^{n+1}\left(\xi_{k}\right)=f_{j}^{n}\left(\xi_{k}\right)-\xi_{k} \frac{\Delta t}{\Delta x}\left(f_{j}^{n}\left(\xi_{k}\right)-\tilde{f}_{R, j-\frac{1}{2}}\left(\xi_{k}\right)\right), \\
f_{j}^{n+1}\left(-\xi_{k}\right)=f_{j}^{n}\left(-\xi_{k}\right)+\xi_{k} \frac{\Delta t}{\Delta x}\left(\tilde{f}_{L, j+\frac{1}{2}}\left(-\xi_{k}\right)-f_{j}^{n}\left(-\xi_{k}\right)\right) .
\end{array}\right.
$$

The values $\tilde{f}_{R, j-\frac{1}{2}}\left(\xi_{k}\right)$ are deduced from the "left" problem,

$$
M\left(\begin{array}{c}
\mathbf{A} \\
\mathbf{B}
\end{array}\right)=\frac{2}{c}\left(\begin{array}{c}
f_{j-1}^{n}(\boldsymbol{\xi}) \\
f_{j}^{n}(-\boldsymbol{\xi})
\end{array}\right)
$$

and the ones $\tilde{f}_{L, j+\frac{1}{2}}\left(-\xi_{k}\right)$, from the "right" problem,

$$
M\left(\begin{array}{c}
\mathbf{A} \\
\mathbf{B}
\end{array}\right)=\frac{2}{c}\left(\begin{array}{c}
f_{j}^{n}(\boldsymbol{\xi}) \\
f_{j+1}^{n}(-\boldsymbol{\xi})
\end{array}\right) .
$$

Remark 1 We stress that the scheme (18) never makes any approximation of the integral collision term by means of a finite summation. Instead, it is the elementary solution itself which is approximated through the ADO method, especially the continuous part of the spectrum. We believe, and numerical evidence supports this idea, that the overall accuracy is improved by this treatment.

Figures 1 and 2 display numerical results for this well-balanced Godunov scheme: we considered a bounded computational domain $x \in[-1,1]$ with reflecting boundary conditions on each side in order to study stabilization as time grows and $\Delta x>0$ is fixed. The value of $\Delta x$ is given by imposing $2^{7}=128$ grid points 

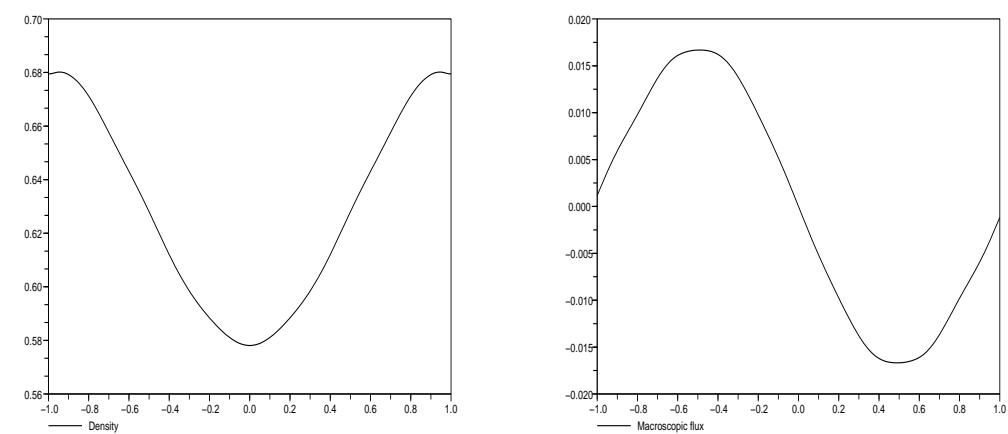

Figure 1: Macroscopic density (left) and flux (right) at time $t=3$.

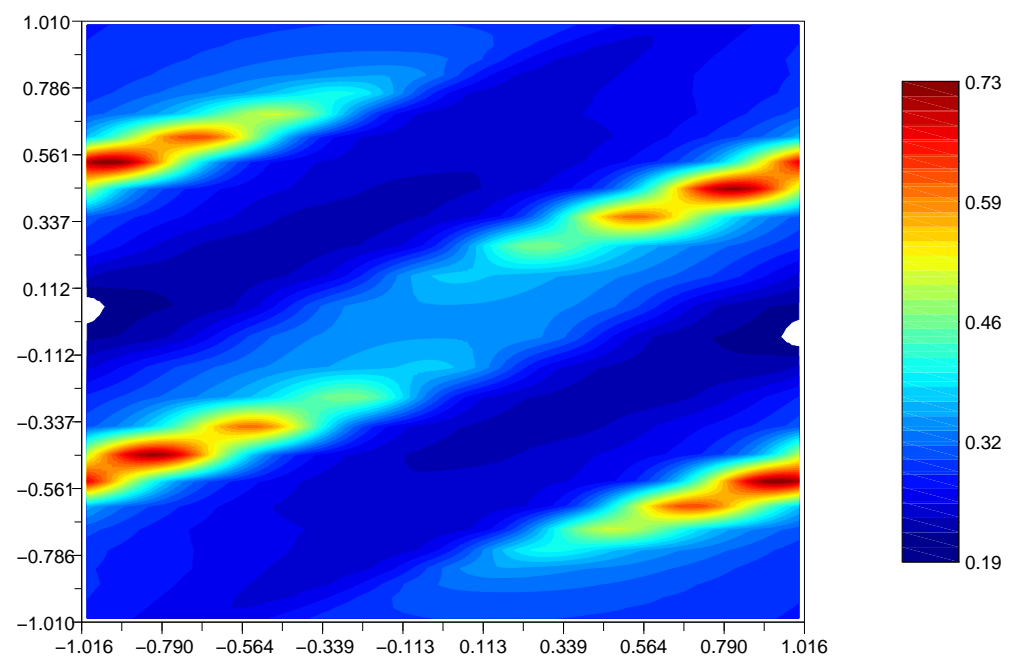

Figure 2: Kinetic density at time $t=3$. 
in the $x$ variable and the time-step $\Delta t$ is deduced from the CFL number of 0.9 . We took $N=15$ grid points for the Gaussian quadrature rule which gives 30 points for gridding the interval $\xi \in[-1,1]$. Initial data consist in 2 bumps:

$$
f^{0}(x, \xi)=10 \exp \left(-20(\xi \mp 0.35)^{2}-50(x \pm 0.35)^{2}\right) .
$$

The parameter $c=0.85$ and we iterate until time $t=3$. The macroscopic density and flux are free from oscillations, so is the kinetic density too when displayed in the $x, \xi$ plane.

\subsection{Conservative case $c=1$ (purely scattering)}

Let us begin by recalling that the equation (1) with $c=1$ has been completely analysed in $\S 3$ of [13] by means of the Laplace transform and the method of elementary solutions. In our numerical context, we follow the Appendix F in [11] and replace the expansion (7) with the one (8) in order to deal with the degeneracy at infinity, $\nu_{0} \rightarrow+\infty$. Clearly, this doesn't affect the pre-processing step dealing with the computation of the eigenmodes vector $\boldsymbol{\nu}$. However, the expression of the ADO solution has to be amended accordingly:

$$
f\left(x, \pm \xi_{k}\right)=\frac{1}{2} E\left(x, \pm \xi_{k}, \nu\right)+\frac{\alpha}{2}+\frac{\beta}{2}\left(x \mp \xi_{k}\right),
$$

where

$$
E(x, \xi, \nu)=\sum_{\ell=1}^{N-1}\left(\frac{A_{\ell} \exp \left(-\left(\frac{\Delta x}{2}+x\right) / \nu_{\ell}\right)}{1-\xi / \nu_{\ell}}+\frac{B_{\ell} \exp \left(-\left(\frac{\Delta x}{2}-x\right) / \nu_{\ell}\right)}{1+\xi / \nu_{\ell}}\right) .
$$

Consequently, the matrix $M$ is deduced from the preceding one (16) only by modifying two of its columns (which are related to the discrete spectrum of the continuous equation):

$$
M_{k, N} \equiv 1, \quad M_{k=1, \ldots, N, 2 N}=-\boldsymbol{\xi}, M_{k=1+N, \ldots, 2 N, 2 N}=\boldsymbol{\xi}+\Delta x .
$$

It is very interesting to relate this modification of $M$ with what has been found in [22] for the 2-velocity model for which there are no "damped modes" (the continuous part of the spectrum). Indeed, in this case, the matrix $M$ reads:

$$
M=\left(\begin{array}{cc}
1 & -1 \\
1 & 1+\Delta x
\end{array}\right)
$$

With the notation of [22], we obtain that:

$$
\alpha=\frac{2\left((1+\Delta x) u_{L}+v_{R}\right)}{2+\Delta x}, \quad \beta=\frac{2\left(-u_{L}+v_{R}\right)}{2+\Delta x} .
$$

Hence, we recover the expressions

$$
\tilde{u}=u_{L}+\frac{\Delta x / 2}{1+\Delta x / 2}\left(v_{R}-u_{L}\right), \quad \tilde{v}=v_{R}-\frac{\Delta x / 2}{1+\Delta x / 2}\left(v_{R}-u_{L}\right),
$$


which means that the jump relations derived in [22] are particular cases of elementary solutions with a discrete spectrum. The present Godunov scheme (18) with its interface values determined either in the dissipative case $c<1$, or in the conservative case $c=1$ is likely to be the most natural generalization of the simple discrete velocity model studied in [22]. On Figures 3 and 4, we display numerical results for the test-case corresponding to the same initial data than in the preceding subsection. The only change is that $c=1$ and the modified $M$ matrix is used for computing the vectors $\mathbf{A}$ and $\mathbf{B}$. Since there is no dissipation, the total mass is preserved and we can therefore observe the time-asymptotic behavior of the WB Godunov scheme, especially the decay of its numerical solution onto the Maxwellian distribution. We aim at observing numerically that, as time passes, the numerical kinetic density becomes less and less varying in the $\xi$ velocity variable. This is actually what happens as can be seen in Figure 5 despite the reflecting boundary conditions in $x= \pm 1$.

\subsection{Comparison with a classical time-splitting scheme}

One may think that a conventional time-splitting scheme can behave equally well since the equation (1) is linear and the ordinary differential equation associated to the collision term can be integrated exactly since the macroscopic density $\varrho$ is invariant along its flow. Indeed, such a scheme would read:

$$
\left\{\begin{array}{l}
f_{j}^{n+\frac{1}{2}}\left(\xi_{k}\right)=f_{j}^{n}\left(\xi_{k}\right)-\xi_{k} \frac{\Delta t}{\Delta x}\left(f_{j}^{n}\left(\xi_{k}\right)-f_{j-1}^{n}\left(\xi_{k}\right)\right), \\
f_{j}^{n+\frac{1}{2}}\left(-\xi_{k}\right)=f_{j}^{n}\left(-\xi_{k}\right)+\xi_{k} \frac{\Delta t}{\Delta x}\left(f_{j+1}^{n}\left(-\xi_{k}\right)-f_{j}^{n}\left(-\xi_{k}\right)\right),
\end{array}\right.
$$

together with:

$$
f_{j}^{n+1}\left( \pm \xi_{k}\right)=\exp (-\Delta t) f_{j}^{n+\frac{1}{2}}\left( \pm \xi_{k}\right)+\frac{c}{2}(1-\exp (-\Delta t)) \varrho_{j}^{n+\frac{1}{2}}
$$

Many variants, like the second order Strang splitting, exist and they probably share the same problem when it comes to long-time behavior. Actually, the first part (20) creates variation in the $\xi$ variable since it separates the particles according to their velocity: in particular, it drives apart left-moving particles from right-moving ones; this is stated rigorously as the so-called dispersion Lemma 2.1 in [37]. Later, comes the relaxation step which projects the resulting kinetic density onto $\varrho$; however, this doesn't compensate for the preceding step. There is no mechanism in this scheme which can efficiently tame the variations in the $\xi$ variable of its numerical kinetic density. This manifests itself through several signs: when considering the decay of the $L^{2}$ time residues,

$$
R^{n}=\left\|\varrho\left(t^{n}, .\right)-\varrho\left(t^{n-1}, .\right)\right\|_{L^{2}}, \quad n \in \mathbb{N}_{*},
$$

one easily sees on the top of Figure 6 that the ones of the time-splitting scheme decrease less quickly and in a more erratic way compared to the ones of the wellbalanced scheme (15). Moreover, by looking at the numerical kinetic densities 

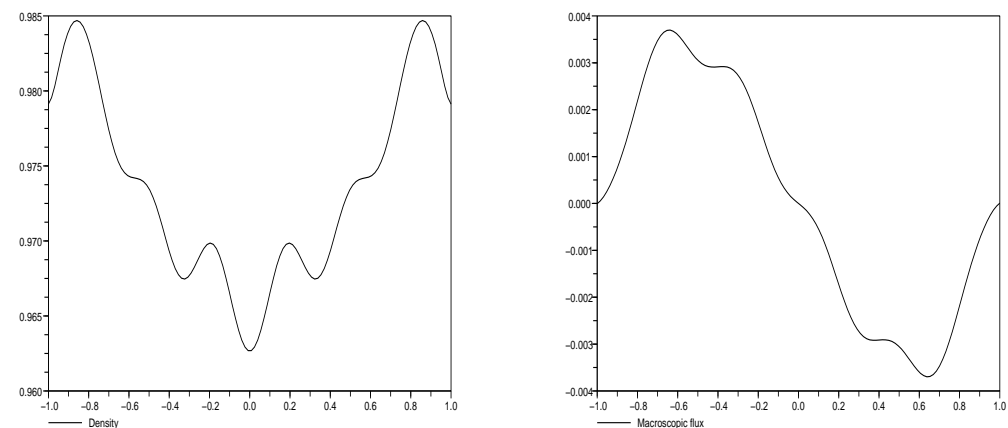

Figure 3: Macroscopic density (left) and flux (right) at time $t=3$.

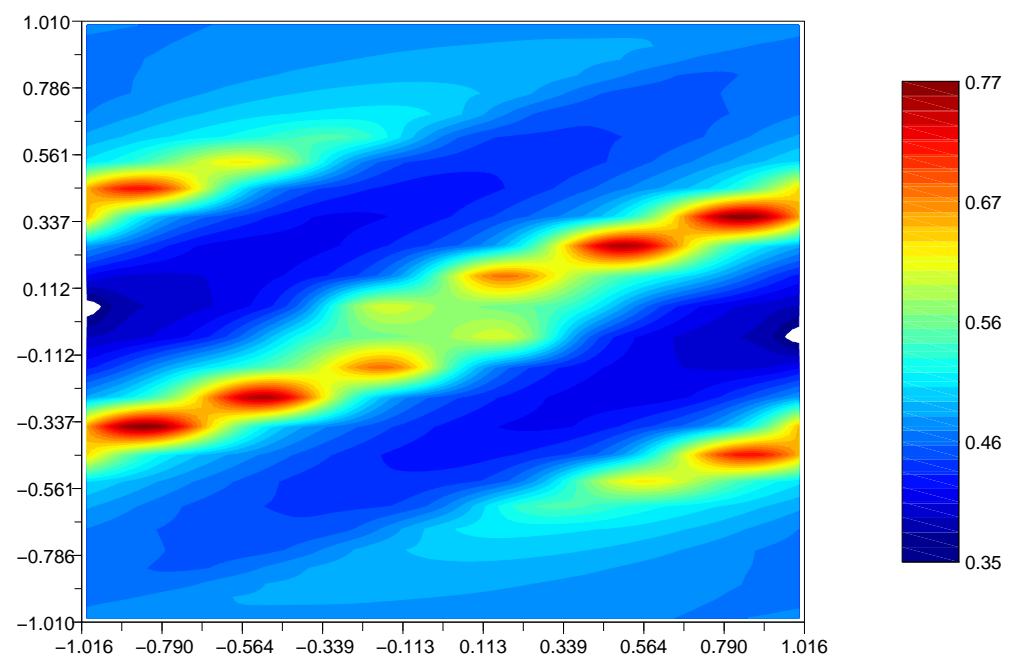

Figure 4: Kinetic density at time $t=3$. 

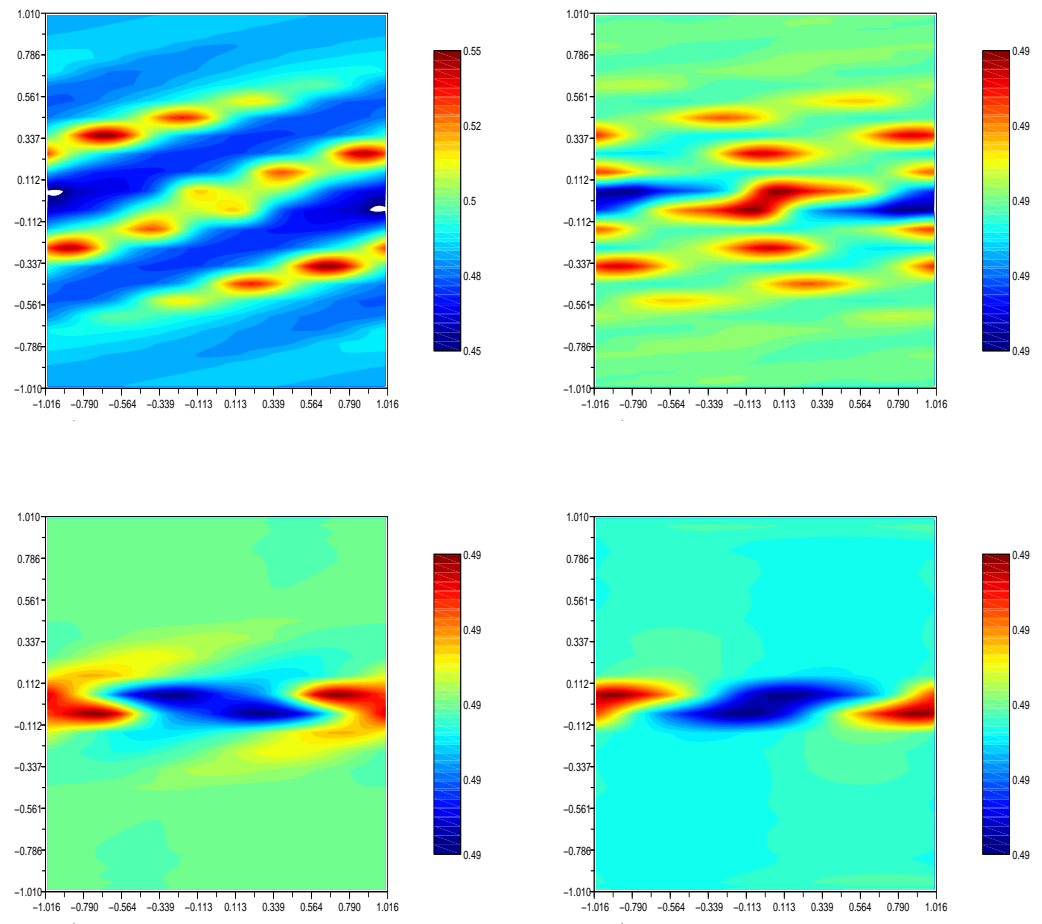

Figure 5: Kinetic densities at $t=5,9.5,25,35$ (left to right, top to bottom).

in the $x, \xi$ plane at later time $t=15$ on Figure 7 , one sees that many ripples still appear on the time-splitting solution w.r.t. the WB as a consequence of the drawback explained formerly. It comes as no surprise that the resulting macroscopic density also displays more variation. Time-splitting schemes have been studied in [20] for a relaxing system in hyperbolic scaling admitting BVbounds according to a $\mathrm{NC}$ formalism related to the one we used presently.

\section{Asymptotic-Preserving for diffusive regime}

In this section, we shall always be concerned with the rescaled problem (2) as we now aim at modifying the Godunov scheme (15) in order to make stable independently of the smallness of $\varepsilon$ and moreover consistent with the limiting diffusion equation (3) as $\varepsilon \rightarrow 0$ with $\Delta x>0$ fixed. 

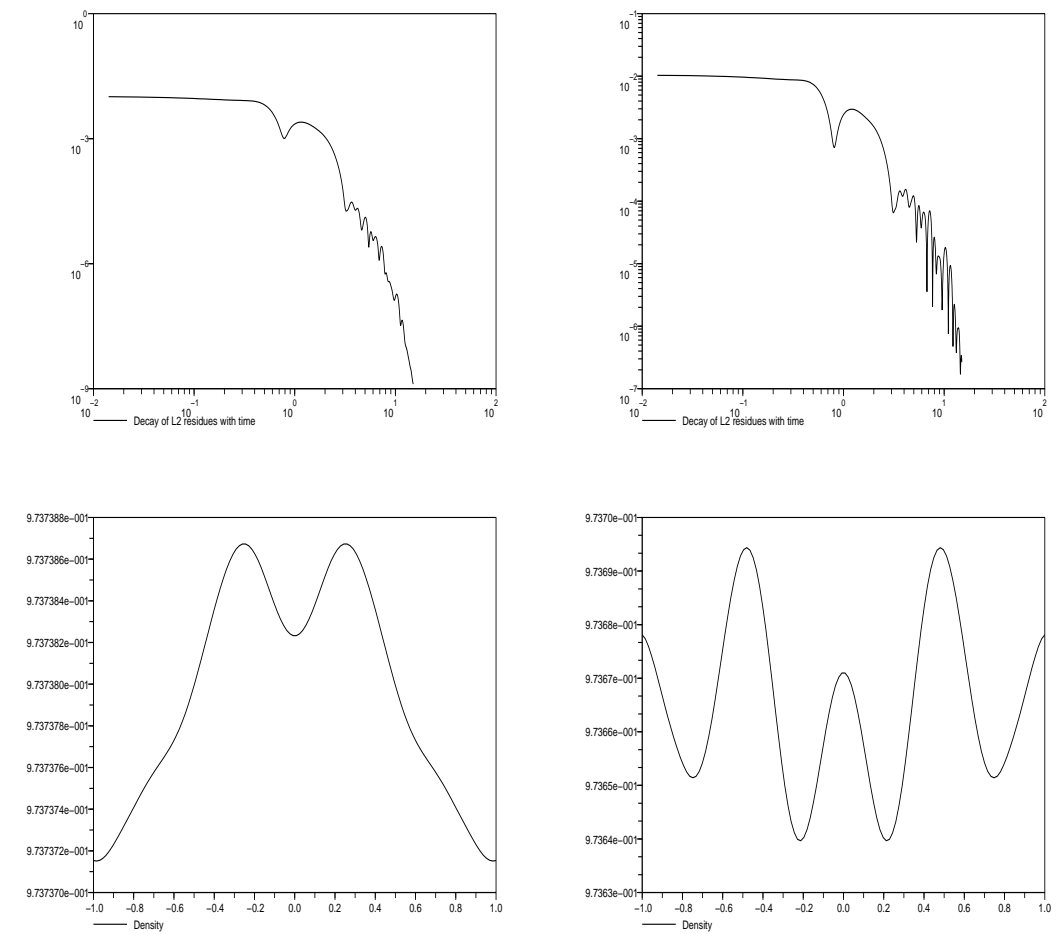

Figure 6: Residues (top) and macroscopic densities (bottom) at time $t=15$.
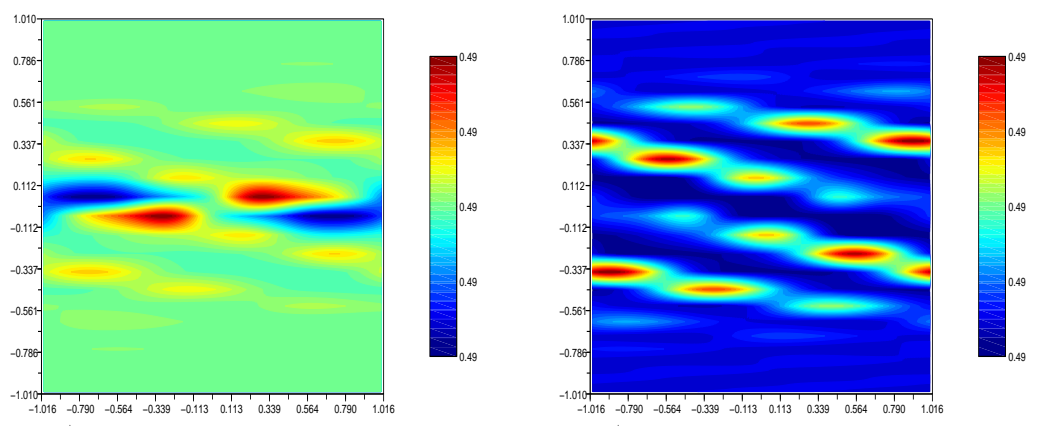

Figure 7: Comparison of kinetic densities at $t=15$ : WB (left) and TS (right). 


\subsection{Splitting between Maxwellian and diffusive fluxes}

To cope with the diffusive scaling in (2), we rewrite the stationary equation,

$$
\xi \partial_{x} f(x, \xi)+f(x, \xi)=\frac{1}{2} \int_{-1}^{1} f\left(x, \xi^{\prime}\right) d \xi^{\prime}, \quad x \in\left(-\frac{\Delta x}{2 \varepsilon}, \frac{\Delta x}{2 \varepsilon}\right),
$$

together the ADO approximation of Case's elementary solution:

$$
f\left(x, \pm \xi_{k}\right)=\frac{1}{2} E\left(x / \varepsilon, \pm \xi_{k}, \nu\right)+\frac{\alpha}{2}+\frac{\beta}{2}\left(\frac{x}{\varepsilon} \mp \xi_{k}\right) .
$$

Thus the $M$ matrix has to be modified accordingly,

$$
M^{\varepsilon}=\left(\begin{array}{cc}
\left(1-\boldsymbol{\xi} \otimes \boldsymbol{\nu}^{-1}\right)^{-1} & \left(1+\boldsymbol{\xi} \otimes \boldsymbol{\nu}^{-1}\right)^{-1} \exp \left(-\frac{\Delta x}{\varepsilon \boldsymbol{\nu}}\right) \\
\left(1+\boldsymbol{\xi} \otimes \boldsymbol{\nu}^{-1}\right)^{-1} \exp \left(-\frac{\Delta x}{\varepsilon \boldsymbol{\nu}}\right) & \left(1-\boldsymbol{\xi} \otimes \boldsymbol{\nu}^{-1}\right)^{-1}
\end{array}\right),
$$

with its two columns reading like:

$$
M_{k, N}^{\varepsilon} \equiv 1, \quad M_{k=1, \ldots, N, 2 N}^{\varepsilon}=-\boldsymbol{\xi}, M_{k=1+N, \ldots, 2 N, 2 N}=\boldsymbol{\xi}+\frac{\Delta x}{\varepsilon} .
$$

The interface values come from the solutions of the linear systems:

$$
M^{\varepsilon}\left(\begin{array}{c}
\mathbf{A} \\
\mathbf{B}
\end{array}\right)=2\left(\begin{array}{c}
f_{j-1}^{n}(\boldsymbol{\xi}) \\
f_{j}^{n}(-\boldsymbol{\xi})
\end{array}\right), \quad M^{\varepsilon}\left(\begin{array}{c}
\mathbf{A} \\
\mathbf{B}
\end{array}\right)=2\left(\begin{array}{c}
f_{j}^{n}(\boldsymbol{\xi}) \\
f_{j+1}^{n}(-\boldsymbol{\xi})
\end{array}\right) .
$$

Looking at (15), it seems far less easy than in $[22,23]$ to separate inside the interface values $\tilde{f}_{L / R, j+\frac{1}{2}}\left(\xi_{k}\right)$ between a Maxwellian $O(1)$ term and a diffusive $O(\varepsilon)$ one as their explicit expression isn't available. However, we can proceed based on the guidelines of the simple linear Goldstein-Taylor model: according to the computation of the previous section, the coefficient $\beta$ in (19) is a good candidate for a diffusive term. Thus, we propose the following decomposition of the ADO interface values:

1. the Maxwellian terms $2 \mathcal{M}_{L}\left(-\xi_{k}\right)=E\left(-\Delta x / \varepsilon, \xi_{k}, \nu\right)+\alpha$ and $2 \mathcal{M}_{R}\left(\xi_{k}\right)=$ $E\left(\Delta x / \varepsilon, \xi_{k}, \nu\right)+\alpha+\beta \frac{\Delta x}{\varepsilon}$ where $E$ contains the "damped modes" expressed with exponential functions which are probably very small when $\varepsilon \ll \Delta x$,

2. the diffusive terms $2 \mathcal{D}_{L}\left(-\xi_{k}\right)=\beta\left|\xi_{k}\right|$ and $2 \mathcal{D}_{R}\left(\xi_{k}\right)=-\beta\left|\xi_{k}\right|$, independent on $\Delta x$, which also have the nice feature of making the coefficient $\frac{1}{3}$ easily appear when the discrete equation on $\varrho_{j}^{n}$ is derived.

By treating implicitly the Maxwellian part and explicitly the diffusive one, the Godunov scheme (15) rewrites in the diffusive scaling as follows:

$$
\left\{\begin{array}{l}
f_{j}^{n+1}\left(\xi_{k}\right)=\left[\frac{f_{j}^{n}\left(\xi_{k}\right)}{1+\frac{\xi_{k} \Delta t}{\varepsilon \Delta x}}+\frac{\frac{\xi_{k} \Delta t}{\varepsilon \Delta x}}{1+\frac{\xi_{k} \Delta t}{\varepsilon \Delta x}} \mathcal{M}_{R, j-\frac{1}{2}}\left(\xi_{k}\right)\right]-\xi_{k}^{2} \frac{\Delta t}{\Delta x} \frac{\beta_{j-\frac{1}{2}}^{n}}{\varepsilon} \\
f_{j}^{n+1}\left(-\xi_{k}\right)=\left[\frac{f_{j}^{n}\left(-\xi_{k}\right)}{1+\frac{\xi_{k} \Delta t}{\varepsilon \Delta x}}+\frac{\frac{\xi_{k} \Delta t}{\varepsilon \Delta x}}{1+\frac{\xi_{k} \Delta t}{\varepsilon \Delta x}} \mathcal{M}_{L, j+\frac{1}{2}}\left(-\xi_{k}\right)\right]+\xi_{k}^{2} \frac{\Delta t}{\Delta x} \frac{\beta_{j+\frac{1}{2}}^{n}}{\varepsilon}
\end{array}\right.
$$


For the time being, we are not able to derive rigorous proofs for the scheme (22) and we shall mainly rely on numerical evidence displayed in the subsection $\S 4.3$. Following [22], we can derive formally the consistency of (22) with (3) assuming that each $f_{j}^{n}\left(\xi_{k}\right)$ is close to Maxwellian. Indeed, (22) rewrites:

$$
\left\{\begin{array}{l}
f_{j}^{n+1}\left(\xi_{k}\right)=f_{j}^{n}\left(\xi_{k}\right)-\frac{\xi_{k} \Delta t}{\varepsilon \Delta x}\left(f_{j}^{n+1}\left(\xi_{k}\right)-\mathcal{M}_{R, j-\frac{1}{2}}\left(\xi_{k}\right)\right)-\xi_{k}^{2} \frac{\Delta t}{\Delta x} \frac{\beta_{j-\frac{1}{2}}^{n}}{\varepsilon}, \\
f_{j}^{n+1}\left(-\xi_{k}\right)=f_{j}^{n}\left(-\xi_{k}\right)+\frac{\xi_{k} \Delta t}{\varepsilon \Delta x}\left(\mathcal{M}_{L, j+\frac{1}{2}}\left(-\xi_{k}\right)-f_{j}^{n+1}\left(-\xi_{k}\right)\right)+\xi_{k}^{2} \frac{\Delta t}{\Delta x} \frac{\beta_{j+\frac{1}{2}}^{n}}{\varepsilon} .
\end{array}\right.
$$

We multiply each equation for $\pm \boldsymbol{\xi}$ by the weights vector $\boldsymbol{\omega}$ and sum up:

$$
\begin{aligned}
& \frac{\varrho_{j}^{n+1}-\varrho_{j}^{n}}{\Delta t}-\frac{1}{3} \frac{\beta_{j+\frac{1}{2}}^{n}-\beta_{j-\frac{1}{2}}^{n}}{\varepsilon \Delta x}= \\
& \sum_{k=1}^{N} \frac{\omega_{k} \xi_{k}}{\varepsilon \Delta x}\left(\left[\mathcal{M}_{R, j-\frac{1}{2}}\left(\xi_{k}\right)+\mathcal{M}_{L, j+\frac{1}{2}}\left(-\xi_{k}\right)\right]-\left[f_{j}^{n+1}\left(\xi_{k}\right)+f_{j}^{n+1}\left(-\xi_{k}\right)\right]\right) .
\end{aligned}
$$

The right-hand side becomes small as $\varepsilon \rightarrow 0$ in the computations shown in $\S 4.3$.

Remark 2 The scheme (22) is hopefully asymptotic-preserving independently of the number of points $N$ chosen in the Gaussian quadrature for the ADO method, and consequently of the number of equations in the hyperbolic system obtained from the discretization in the $\xi$ variable.

Once again, it is of interest to observe how behaves our Maxwellian/diffusive splitting on the simple Goldstein-Taylor model; the matrix $M^{\varepsilon}$ simplifies to

$$
M^{\varepsilon}=\left(\begin{array}{cc}
1 & -1 \\
1 & 1+\frac{\Delta x}{\varepsilon}
\end{array}\right)
$$

and from the equation $M^{\varepsilon}(\alpha \beta)^{T}=2\left(u_{L} v_{R}\right)^{T}$ solved at any interface $x_{j+\frac{1}{2}}$, $j \in \mathbb{Z}$, of the computational domain, it comes:

$$
\beta=\frac{-2}{2+\Delta x / \varepsilon}\left(u_{L}-v_{R}\right)=\frac{-\varepsilon}{\varepsilon+\Delta x / 2}\left(u_{L}-v_{R}\right) .
$$

And this is exactly the right term yielding the correct asymptotic diffusion when multiplied by $\Delta t / \varepsilon \Delta x$ with $\varepsilon \rightarrow 0$; moreover, this type of value would lead to a centered discretization of the second derivative $\frac{1}{3} \partial_{x x} \varrho$ in (23). Thus we have every reason to believe that the aforementioned decomposition still works in the general case where $2 N$ velocities $\pm \boldsymbol{\xi}$ are considered.

Proposition 1 Let $f$ be defined as (21); if $c=1$, there holds:

$$
\forall x \in\left(-\frac{\Delta x}{2}, \frac{\Delta x}{2}\right), \quad \int_{-1}^{1} \xi f(x, \xi) d \xi \equiv-\frac{\beta}{3} .
$$


Proof. It proceeds by approximately integrating the piecewise constant function in (21) according to the quadrature rule $\boldsymbol{\omega}, \boldsymbol{\xi}$ introduced in (9):

$$
\int_{-1}^{1} \xi f(x, \xi) d \xi=\sum_{k=1}^{N} \omega_{k} \xi_{k}\left[f\left(x, \xi_{k}\right)-f\left(x,-\xi_{k}\right)\right]
$$

One part of the integral clearly vanishes because $\xi \mapsto(\alpha+\beta x / \varepsilon) \xi$ is odd on $(-1,1)$. Concerning the exponential terms, we leave the part depending on $x$ and recall the normalization condition $(14)$ in order to compute for any $\nu \in(-1,1)$ :

$$
\begin{aligned}
\sum_{k=1}^{N} \omega_{k} \xi_{k}\left(\varphi\left(\nu, \xi_{k}\right)-\varphi\left(\nu,-\xi_{k}\right)\right) & =\frac{c}{2} \sum_{k=1}^{N} \omega_{k}\left(\frac{\xi_{k}}{1-\xi_{k} / \nu}-\frac{\xi_{k}}{1+\xi_{k} / \nu}\right) \\
& =-\frac{c \nu}{2} \sum_{k=1}^{N} \omega_{k}\left(2-\frac{1}{1-\xi_{k} / \nu}-\frac{1}{1+\xi_{k} / \nu}\right) \\
& =-\frac{c \nu}{2}\left(2-\frac{2}{c} \sum_{k=1}^{N} \omega_{k}\left[\varphi\left(\nu, \xi_{k}\right)+\varphi\left(\nu,-\xi_{k}\right)\right]\right) .
\end{aligned}
$$

At this point, inserting $c=1$ ensures that this term vanishes. Thus remains:

$$
\sum_{k=1}^{N} \omega_{k} \xi_{k}\left[f\left(x, \xi_{k}\right)-f\left(x,-\xi_{k}\right)\right]=-\frac{\beta}{2} \sum_{k=1}^{N} \omega_{k}\left(2 \xi_{k}^{2}\right)=-\frac{\beta}{3} .
$$

Proposition 1 is somehow a restatement of equation (40) in [4]; it explains why the diffusive fluxes appearing in the left-hand side of (23) are correct for $c=1$ and $\varepsilon$ small enough. The fact that neither $\Delta x$ nor $\varepsilon$ show up in the expression of these fluxes ensures the AP property and the consistency of (22) with (3).

\subsection{Relation with a previous AP scheme}
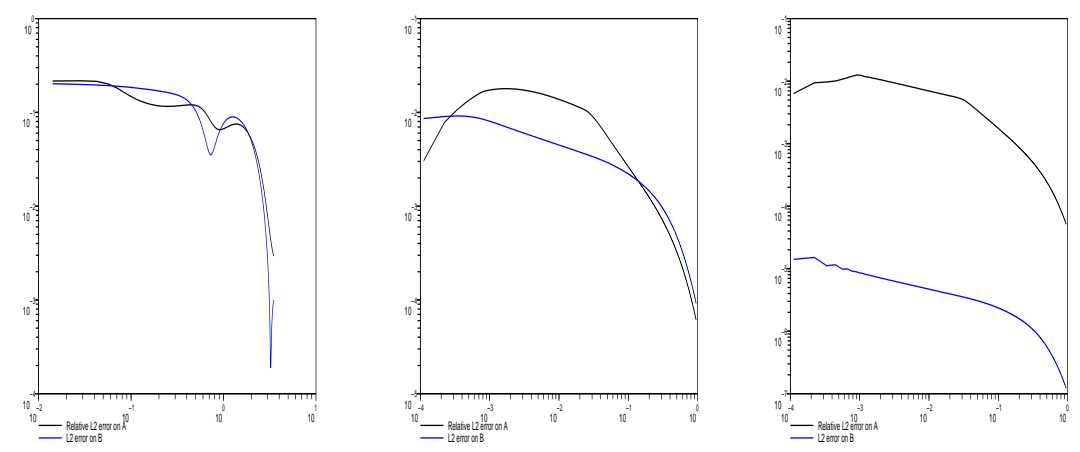

Figure 8: Errors on approximating $\alpha, \beta$ for various $\varepsilon$ with (24) and (25).

In the previous decomposition, we included in the Maxwellian part the terms of the order of $\exp (-\Delta x / \varepsilon \boldsymbol{\nu})$ despite the fact they decrease very quickly with $\varepsilon$. It is therefore very tempting to cancel them when deriving the scheme (22); 
a first consequence of this assumption is to get Maxwellian terms independent on $\xi_{k}$. However, there is another interesting one: on Figure 8, we display the numerical the behavior of the following approximations for the values $\alpha$ and $\beta$,

$$
\alpha \simeq \frac{2}{2+\Delta x}\left((1+\Delta x) \sum_{k=1}^{N} \omega_{k} f_{\text {left }}\left(\xi_{k}\right)+\sum_{k=1}^{N} \omega_{k} f_{\text {right }}\left(-\xi_{k}\right)\right)
$$

and

$$
\beta \simeq-\frac{2}{2+\Delta x}\left(\sum_{k=1}^{N} \omega_{k} \xi_{k}\left(f_{\text {left }}\left(\xi_{k}\right)-f_{\text {right }}\left(-\xi_{k}\right)\right)\right)
$$

for $\varepsilon=1, \varepsilon=0.01, \varepsilon=0.00001$ as a function of time. Of course, for $\varepsilon \ll \Delta x$, the value $\Delta x$ is replaced by $\Delta x / \varepsilon$ as we pass from the scheme (15) to the AP one (22). One sees that these simple approximations improve when the kinetic density becomes slowly-varying in the velocity variable $\xi$. These values of $\alpha$ and $\beta$ mean that two reasonable assumptions are made: one, the kinetic density is close to its Maxwellian distribution and two, the exponential terms corresponding to the continuous spectrum are negligible. Then it can be checked that, in the notation of (17), the following jump relations hold:

$$
\begin{aligned}
\tilde{f}_{L}\left(-\xi_{k}\right)= & \frac{1}{2}\left(\alpha+\beta \xi_{k}\right) \\
= & \sum_{\ell=1}^{N} \omega_{\ell} f_{\text {left }}\left(\xi_{k}\right)-\frac{1}{2+\Delta x / \varepsilon} \sum_{\ell=1}^{N} \omega_{\ell}\left(f_{\text {left }}\left(\xi_{k}\right)-f_{\text {right }}\left(-\xi_{k}\right)\right) \\
& -\frac{\xi_{k}}{2+\Delta x / \varepsilon} \sum_{\ell=1}^{N} \omega_{\ell} \xi_{\ell}\left(f_{\text {left }}\left(\xi_{k}\right)-f_{\text {right }}\left(-\xi_{k}\right)\right), \\
= & \sum_{\ell=1}^{N} \omega_{\ell} f_{\text {left }}\left(\xi_{k}\right)-\varepsilon \sum_{\ell=1}^{N} \frac{1+\xi_{k} \xi_{\ell}}{2 \varepsilon+\Delta x} \omega_{\ell}\left(f_{\text {left }}\left(\xi_{k}\right)-f_{\text {right }}\left(-\xi_{k}\right)\right) \\
\simeq & \sum_{\ell=1}^{N} \omega_{\ell} f_{\text {left }}\left(\xi_{k}\right)-\frac{2 \varepsilon \xi_{k}}{2 \varepsilon+\Delta x} \sum_{\ell=1}^{N} \omega_{\ell}\left(f_{\text {left }}\left(\xi_{k}\right)-f_{\text {right }}\left(-\xi_{k}\right)\right)
\end{aligned}
$$

and

$$
\begin{aligned}
\tilde{f}_{R}\left(\xi_{k}\right)= & \frac{1}{2}\left(\alpha+\beta \Delta x / \varepsilon-\beta \xi_{k}\right) \\
= & \sum_{\ell=1}^{N} \omega_{\ell} f_{\text {right }}\left(-\xi_{k}\right)+\frac{1+\Delta x / \varepsilon}{2+\Delta x / \varepsilon} \sum_{\ell=1}^{N} \omega_{\ell}\left(f_{\text {left }}\left(\xi_{k}\right)-f_{\text {right }}\left(-\xi_{k}\right)\right) \\
& +\frac{\xi_{k}-\Delta x / \varepsilon}{2+\Delta x / \varepsilon} \sum_{\ell=1}^{N} \omega_{\ell} \xi_{\ell}\left(f_{\text {left }}\left(\xi_{k}\right)-f_{\text {right }}\left(-\xi_{k}\right)\right) \\
= & \sum_{\ell=1}^{N} \omega_{\ell} f_{\text {right }}\left(-\xi_{k}\right)+\sum_{\ell=1}^{N} \omega_{\ell} \frac{\left(1-\xi_{\ell}\right) \Delta x}{2 \varepsilon+\Delta x}\left(f_{\text {left }}\left(\xi_{k}\right)-f_{\text {right }}\left(-\xi_{k}\right)\right) \\
& +\varepsilon \sum_{\ell=1}^{N} \omega_{\ell} \frac{1+\xi_{k} \xi_{\ell}}{2 \varepsilon+\Delta x}\left(f_{\text {left }}\left(\xi_{k}\right)-f_{\text {right }}\left(-\xi_{k}\right)\right) \\
\simeq & \sum_{\ell=1}^{N} \omega_{\ell} f_{\text {right }}\left(-\xi_{k}\right)+\frac{2 \varepsilon \xi_{k}}{2 \varepsilon+\Delta x} \sum_{\ell=1}^{N} \omega_{\ell}\left(f_{\text {left }}\left(\xi_{k}\right)-f_{\text {right }}\left(-\xi_{k}\right)\right)
\end{aligned}
$$


And this matches the results in [24]: the simplifications in the macroscopic fluxes follow the computations in pp. 234-236. Thus, by treating implicitly the stiff terms, we obtain the equations of the scheme (34) with the integral terms replaced by finite sums involving the Gaussian quadrature rule:

$$
\left\{\begin{array}{r}
f_{j}^{n+1}\left(\xi_{k}\right)=f_{j}^{n}\left(\xi_{k}\right)-\frac{\xi_{k} \Delta t}{\varepsilon \Delta x}\left(f_{j}^{n+1}\left(\xi_{k}\right)-\sum_{\ell=1}^{N} \omega_{\ell} f_{j}^{n+1}\left(-\xi_{k}\right)\right) \\
+\frac{2 \xi_{k}^{2} \Delta t}{\Delta x(2 \varepsilon+\Delta x)} \sum_{\ell=1}^{N} \omega_{\ell}\left(f_{j-1}^{n}\left(\xi_{k}\right)-f_{j}^{n}\left(-\xi_{k}\right)\right) \\
f_{j}^{n+1}\left(-\xi_{k}\right)=f_{j}^{n}\left(-\xi_{k}\right)+\frac{\xi_{k} \Delta t}{\varepsilon \Delta x}\left(\sum_{\ell=1}^{N} \omega_{\ell} f_{j}^{n+1}\left(\xi_{k}\right)-f_{j}^{n+1}\left(-\xi_{k}\right)\right) \\
-\frac{2 \xi_{k}^{2} \Delta t}{\Delta x(2 \varepsilon+\Delta x)} \sum_{\ell=1}^{N} \omega_{\ell}\left(f_{j}^{n}\left(\xi_{k}\right)-f_{j+1}^{n}\left(-\xi_{k}\right)\right) .
\end{array}\right.
$$

We can exploit the rigorous stability results from [24] at least in this special case where drastic simplifications have been made in the ADO approximate solution assuming that the kinetic density is very close to Maxwellian. This also provides us with another manner of deriving the AP schemes studied in this earlier paper.

Remark 3 A big difference between the present work and the former one [24] is that, despite considering the numerical approximation of the same problems (1) and (2), the treatment of the stationary equations is quite different. Indeed, in [24], we approximated this steady-state problem by finite differences; in sharp contrast, we treat it exactly by means of the method of elementary solutions, and we approximate only the continuous part of the spectrum by means of a finite sum with a Gaussian quadrature rule. So the WB scheme (15) can be considered as a potentially much sharper version of the one proposed earlier in §2 of [24].

\subsection{Numerical results}

We now present some numerical results from the scheme (22) in the parabolic scaling (2) with the "nearly Maxwellian" initial data:

$$
\left\{\begin{array}{l}
f^{0}(x, \xi)=\exp \left(-\varepsilon\left(\xi^{2}-x^{2}\right)\right) \chi_{x \in[-1 / 3,1 / 3]}, \\
f^{0}(x, \xi)=\exp \left(-\varepsilon \xi^{2}-x^{2}\right) \chi_{x \in[-1 / 3,1 / 3]}
\end{array} \quad x \in[-1,1]\right.
$$

where $\chi_{A}$ stands for the indicator function of a set $A$. The parabolic CFL condition has been used, namely $\Delta t=0.45 \Delta x^{2}$, we took again $N=15$ and 127 points to grid the $x$ interval. Tests have been carried out up to $t=0.05$ with $\varepsilon=0.01$ and $\varepsilon=0.00001$. For this smaller value of $\varepsilon$, we display on Figure 9 the macroscopic density $\varrho$ and the flux $J$ obtained out of the kinetic density $f_{j}^{n}( \pm \xi)$ computed with (22); especially, the density is compared to the direct solution of the equation (3) computed by means of a standard centered scheme. The consistency can be considered satisfactory. On Figure 10, we display the kinetic distribution for $\varepsilon=0.01$ at time $t=0.05$; clearly, it can be considered as "practically Maxwellian" even if $\varepsilon$ is snaller but still of the order of $\Delta x$. 

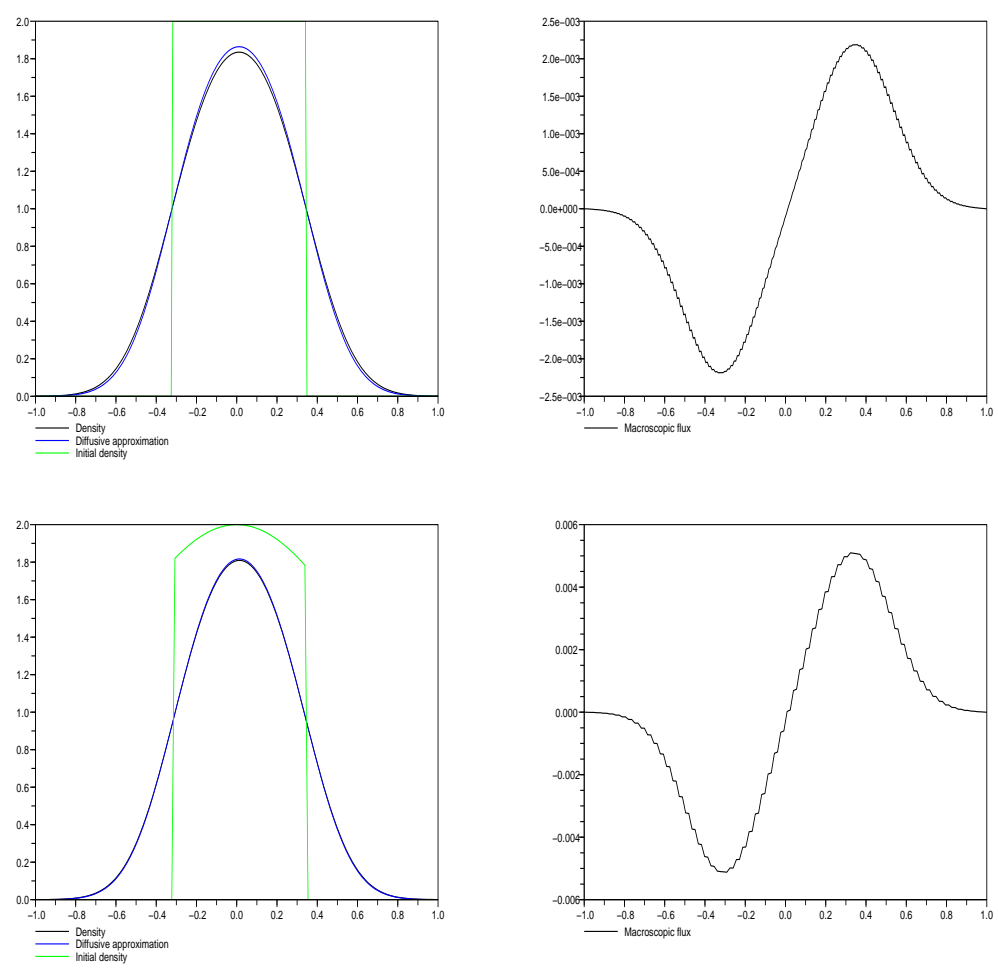

Figure 9: Macroscopic density (left) and flux (right) at time $t=0.05$ for $\varepsilon \ll \Delta x$.

\section{Conclusion and outlook}

An original well-balanced Godunov scheme for the linear kinetic equation (1) has been presented and its numerical results have been compared to the ones generated by a more classical time-splitting discretization. Moreover, it has been shown that it is possible, like in simpler 2-velocities models, to reformulate the scheme in order to make it asymptotic-preserving in the diffusive scaling (2), thus displaying consistency with the limiting diffusion equation (3) independently of the grid parameters as soon as the usual parabolic CFL restriction is met. This approach is by no means limited to this particular radiative transfer equation; namely, 2 main extensions emerge rather spontaneously:

1. more elaborate kinetic models: one can choose to treat any collision operator for which the formalism of Case's elementary solutions can be applied. This class contains for instance the BGK models studied by Cercignani $[12,13,14]$ and treated numerically in the stationary regime by Siewert et al. [4]. Another very interesting direction is to treat Boltzmann equations for charged particles: in this case, one has to follow the results by Dalitz 


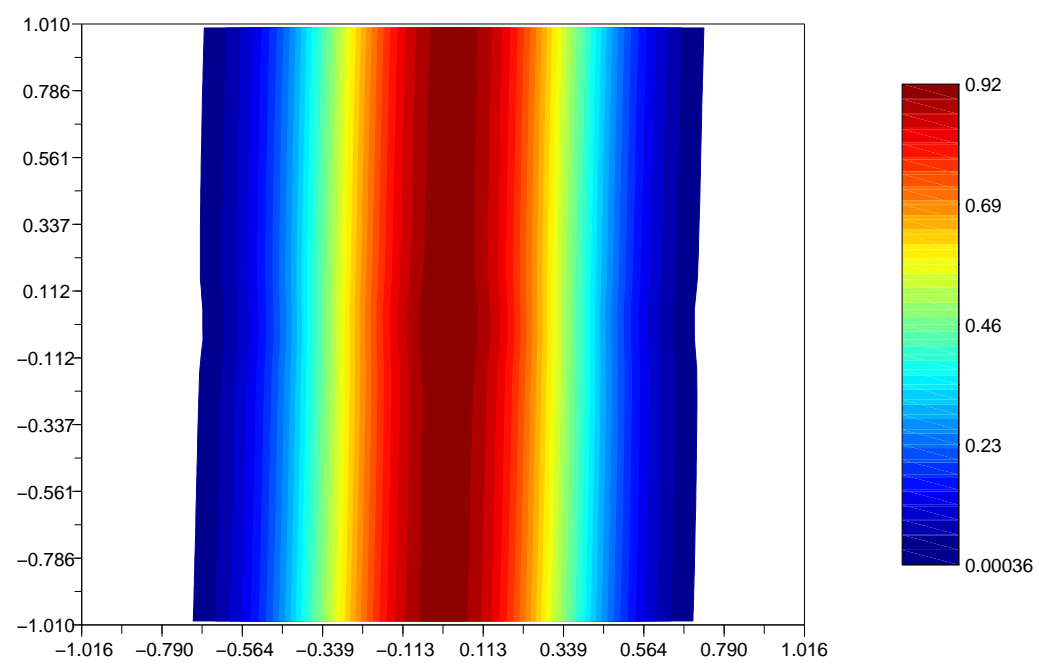

Figure 10: Perturbed Maxwellian kinetic density at time $t=0.05$ for $\varepsilon \simeq \Delta x$.

$[16,18]$ where the elementary solutions are derived in several meaningful situations. It is reasonable to believe that these solutions can handle an unsteady electric field ruled by the Poisson equation in the same manner than the well-balanced scheme in [21] is stable in the presence of the diffusion equation for the chemoattractant substance.

2. numerical coupling between a kinetic and a diffusive region in the computational domain: even if it looks easy to couple the simple schemes derived in [22] (as they are practically the 2 versions of the same discretization), the result isn't satisfactory because mass gets lost at the junction as long as the kinetic density is not Maxwellian in the kinetic region. It would therefore be interesting to fix this issue, as it has been done in [45] for stationary problems.

We close this text metioning that there exists other approaches to numerical calculations in radiative transfer, see for instance [19, 31, 39, 41]. There exists an alternative to Case/ADO method to compute stationary solutions, namely the iterative scheme proposed by Bobylev and Struckmeier [7].

\section{References}

[1] D. Amadori, L. Gosse, G. Guerra, Global BV entropy solutions and uniqueness for hyperbolic systems of balance laws, Arch. Rational Mech. Anal. 162 
(2002) 327-366.

[2] A. Arnold, J.A. Carrillo, M.D. Tidriri, Large-time behavior of discrete equations with non-symmetric interactions, Math. Mod. Meth. in Appl. Sci. 12 (2002) 1555-1564.

[3] C. Bardos, F. Golse, B. Perthame, R. Sentis, The nonaccretive radiative transfer equations: Existence of solutions and Rosseland approximation, J. Funct. Anal. 77 (1988) 434-460.

[4] L.B. Barichello, M. Camargo, P. Rodrigues and C.E. Siewert, Unified Solutions to Classical Flow Problems Based on the BGK Model, ZAMP 52 (2001) 517-534.

[5] L.B. Barichello C.E. Siewert, A discrete-ordinates solution for a non-grey model with complete frequency redistribution, JQSRT 62 (1999) 665-675

[6] Ch. Berthon, P. Charrier, B. Dubroca, An asymptotic preserving relaxation scheme for a moment model of radiative transfer, Comptes rendus Math. 344 (2007) 467-472.

[7] A.V. Bobylev, J Struckmeier, Numerical simulation of the stationary onedimensional Boltzmann equation by particle methods, European Journal of Mechanics, B/Fluids. 15 (1996) 103-118.

[8] Ch. Buet, B. Despres, Asymptotic-preserving and positive schemes for radiation hydrodynamics, J. Comp. Phys. 215 (2006) 717-740.

[9] P. Cargo, G. Samba, Resolution of the time dependent $P_{n}$ equations by a Godunov type scheme having the diffusion limit ESAIM: Math. Model. Numer. Anal. (M2AN) 44 (2010) 1193-1224.

[10] Kenneth M. Case, Elementary solutions of the transport equation and their applications, Ann. Physics 9 (1960) 1-23.

[11] K.M. Case, P.F. Zweifel, Linear transport theory, Addison-Wesley series in nuclear engineering (1967).

[12] C. Cercignani, The method of elementary solutions for kinetic models with velocity dependent collision frequency, Ann. Physics 40 (1966) 469.

[13] C. Cercignani, Solution of a linearized kinetic model for an ultrarelativistic gas, J. Stat. Phys. 42 (1986) 601-620.

[14] C. Cercignani, The Boltzmann equation and its applications, Applied Mathematical Sciences, 67. Springer-Verlag, New York, 1988.

[15] J.J.M. Cuppen, A divide-and-conquer method for the symmetric tridiagonal eigenproblem, Numerische Math. 36 (1981) 177-195. 
[16] Ch. Dalitz, Half-space problem of the Boltzmann equation for charged particles, J. Stat. Phys. 88. (1997) 129-144.

[17] R.C. DeBarros, E. Larsen A numerical method for one-group slab-geometry discrete ordinates problem with no spatial truncation error, Nucl. Sc. Eng. 104 (1990) 199-208.

[18] E. DeGroot, Ch. Dalitz, Exact solution for a boundary value problem in semiconductor kinetic theory, J. Math. Phys. 38 (1997) 4629-4643.

[19] Per Edström, A Fast and Stable Solution Method for the Radiative Transfer Problem, SIAM Review 47 (2005) 447-468.

[20] L. Gosse, Time-splitting schemes and measure source terms for a quasilinear relaxing system, Math. Models Methods Appl. Sci. 13 (2003) 1081-1101.

[21] L. Gosse, Asymptotic-Preserving and Well-Balanced scheme for the $1 D$ Cattaneo model of chemotaxis movement in both hyperbolic and diffusive regimes, submitted to SIAM J. Numer. Anal.

[22] L. Gosse, G. Toscani, An asymptotic-preserving well-balanced scheme for the hyperbolic heat equations, C.R. Math. Acad. Sci. Paris 334 (2002) 337342 .

[23] L. Gosse, G. Toscani, Space localization and well-balanced scheme for discrete kinetic models in diffusive regimes, SIAM J. Numer. Anal. 41 (2003) 641-658.

[24] L. Gosse, G. Toscani, Asymptotic-preserving 83 well-balanced schemes for radiative transfer and the Rosseland approximation, Numerische Math. 98 (2004)

[25] J. Greenberg, A.Y. LeRoux, A well balanced scheme for the numerical processing of source terms in hyperbolic equations, SIAM J. Numer. Anal. 33 (1996) $1-16$.

[26] W. Greenberg, C.V.M. Van der Mee, P.F. Zweifel, Generalized kinetic equations, Integr. Equa. Oper. Theory 7 (1984) 60-95.

[27] F. Guarguaglini, C. Mascia, R. Natalini, M. Ribot, Global stability of constant states and qualitative behavior of solutions to a one dimensional hyperbolic model of chemotaxis, Discrete Contin. Dyn. Syst. Ser. B 12 (2009) 39-76.

[28] C.D. Hauck, R.B. Lowrie, Temporal regularization of the $P_{N}$ equations, SIAM: Math. Model. Simul. 7 (2009) 1497-1524.

[29] E. Isaacson, B. Temple, Convergence of the $2 \times 2$ Godunov method for a general resonant nonlinear balance law, SIAM J. Appl. Math. 55 (1995) $625-640$ 
[30] Shi Jin, David Levermore, The discrete-ordinate method in diffusive regimes, Transp. Theor. Stat. Phys. 20 (1991) 413 - 439.

[31] S. Jin, L. Pareschi, G. Toscani, Uniformly accurate diffusive relaxation schemes for multiscale transport equations, SIAM J. Numer. Anal. 38 (2000) 913-936.

[32] S. Jin, M. Tang, H. Han, On a uniformly second order numerical method for the one-dimensional discrete-ordinate transport equation and its diffusion limit with interface, Networks Heter. Media 4 (2009) 35-65.

[33] A. Klar, An asymptotic induced scheme for nonstationary transport equations in the diffusive limit, SIAM J. Numer. Anal. 35 (1998) 1073-1094

[34] Tomaž Klinc, On completeness of eigenfunctions of the one-speed transport equation, Commun. Math. Phys. 41 (1975) 273-279.

[35] K. Latrach, B. Lods, Regularity and time asymptotic behaviour of solutions to transport equations, Transp. Theory Stat. Phys. 30 (2001) 617-639.

[36] Ph. LeFloch, A.E. Tzavaras, Representation of weak limits and definition of nonconservative products, SIAM J. Math. Anal. 30 (1999), 1309 - 1342.

[37] B. Perthame, Mathematical tools for kinetic equations, Bull. Amer. Math. Soc. 41 (2004) 205-244.

[38] M. Portilheiro, A.E. Tzavaras, Hydrodynamic limits for kinetic equations and the diffusive approximation of radiative transport for acoustic waves, Trans. Amer. Math. Soc. 359 (2007), 529-565.

[39] B. Rutily, J. Bergeat, The solution of the transfer equation in an homogeneous isotropically scattering plane-parallel medium, JQSRT 52 (1994) 857885 .

[40] C.E. Siewert, S.J. Wright Efficient eigenvalue calculations in radiative transfer, J. Quant. Spectro. Radiat. Transf. (1999) 685-688.

[41] K. Stamnes, S.-Chee Tsay, W. Wiscombe, K. Jayaweera Numerically stable algorithm for discrete-ordinate method radiative transfer in multiple scattering and emitting layered media, Applied Optics 27 (1988) 2502-2509.

[42] C. Van der Mee, Exponentially dichotomous operators and applications Birkhäuser (2008) (ISBN 203764387319).

[43] C. Van der Mee, C.E. Siewert On unbounded eigenvalues in transport theory, ZAMP 34 (1983) 556-561.

[44] B. Wendroff, On the convergence of the discrete ordinate method, J. Soc. Indust. Appl. Math. 8 (1960) 508-513 
[45] X. Yang, F. Golse, Z. Huang, S. Jin, Numerical study of a domain decomposition method for a two-scale linear transport equation, Networks Heter. Media 1 (2006) 149-166. 